\title{
Mining geriatric assessment data for in-patient fall prediction models and high-risk subgroups
}

\author{
Michael Marschollek ${ }^{1 *+}$, Mehmet Gövercin ${ }^{3 \dagger}$, Stefan Rust ${ }^{1 \dagger}$, Matthias Gietzelt ${ }^{2 \dagger}$, Mareike Schulze ${ }^{1 \dagger}$, \\ Klaus-Hendrik Wolf ${ }^{2 \dagger}$ and Elisabeth Steinhagen-Thiessen ${ }^{3+}$
}

\begin{abstract}
Background: Hospital in-patient falls constitute a prominent problem in terms of costs and consequences. Geriatric institutions are most often affected, and common screening tools cannot predict in-patient falls consistently. Our objectives are to derive comprehensible fall risk classification models from a large data set of geriatric in-patients' assessment data and to evaluate their predictive performance (aim\#1), and to identify high-risk subgroups from the data (aim\#2).

Methods: A data set of $n=5,176$ single in-patient episodes covering 1.5 years of admissions to a geriatric hospital were extracted from the hospital's data base and matched with fall incident reports ( $n=493$ ). A classification tree model was induced using the C4.5 algorithm as well as a logistic regression model, and their predictive performance was evaluated. Furthermore, high-risk subgroups were identified from extracted classification rules with a support of more than 100 instances.

Results: The classification tree model showed an overall classification accuracy of $66 \%$, with a sensitivity of $55.4 \%$, a specificity of $67.1 \%$, positive and negative predictive values of $15 \%$ resp. $93.5 \%$. Five high-risk groups were identified, defined by high age, low Barthel index, cognitive impairment, multi-medication and co-morbidity.

Conclusions: Our results show that a little more than half of the fallers may be identified correctly by our model, but the positive predictive value is too low to be applicable. Non-fallers, on the other hand, may be sorted out with the model quite well. The high-risk subgroups and the risk factors identified (age, low ADL score, cognitive impairment, institutionalization, polypharmacy and co-morbidity) reflect domain knowledge and may be used to screen certain subgroups of patients with a high risk of falling. Classification models derived from a large data set using data mining methods can compete with current dedicated fall risk screening tools, yet lack diagnostic precision. High-risk subgroups may be identified automatically from existing geriatric assessment data, especially when combined with domain knowledge in a hybrid classification model. Further work is necessary to validate our approach in a controlled prospective setting.
\end{abstract}

Keywords: Accidental falls, Geriatric assessment, Data mining

\section{Background}

Falls and their consequences are a well-known and urgent problem in our ageing population. It is known that geriatric in-patients exhibit the highest fall incidence among institutionalized persons, ranging from 6.3 to $7.2 \%$ within a period of two weeks [1]. About $20-30 \%$

\footnotetext{
* Correspondence: Michael.Marschollek@plri.de

† Contributed equally

${ }^{1}$ Peter L. Reichertz Institute for Medical Informatics, University of Braunschweig - Institute of Technology and Hanover Medical School, CarlNeuberg-Str. 1, 30625 Hanover, Germany

Full list of author information is available at the end of the article
}

of falls result in injuries that need medical intervention $[1,2]$, among which $3-5 \%$ are fractures [2,3]. Apart from the personal consequences of fall events, such as injuries leading to lasting disability and loss of independence or psychological effects such as the post-fall-syndrome [4], they also have economical implications for the health system in general, and for hospitals in particular. The annual costs of falls in the U.S. have been estimated at 19.2\$ billion [5].

In consequence, many assessment tools and risk scales have been developed in order to identify in-patients

\section{Biomed Central}


with a potential fall risk, with the aim to apply timely targeted preventive measures to avoid these events in the first place. Gates reports on 29 different assessment tools, among these e.g. the widely-used Performance-Oriented Mobility Assessment (POMA) by Tinetti [6], and concludes that no explicit recommendation may be given for any single test or scale [7]. Oliver et al. - authors of the St. Thomas Risk Assessment Tool in Falling Elderly In-patients (STRATIFY) [8] - systematically review prospective studies with different assessment scales and conclude that none of these is able to identify a high percentage of fallers correctly [9]. Similar results have been reported by Kim et al. [10]. Most of the available fall risk assessment scales are based on experiential knowledge. The wide-spread use of electronic documentation systems makes large amounts of patient data available. This data can be used to extract information automatically, employing methods of machine learning and data mining, e.g. to generate classification models or to identify specific subgroups of patients who have a high mortality risk [11].

The aim of our research work for this paper is to employ a data mining approach in order

- To derive comprehensible fall risk classification models from a large data set of geriatric in-patients' assessment data and to evaluate their predictive performance (aim\#1), and

- To identify subgroups within a geriatric in-patient population who have a high fall risk (aim\#2).

\section{Methods \\ Study data set}

The study data set comprises all in-patient episodes from July 2006 to December 2007 at the Evangelisches Geriatriezentrum Berlin $g G m b H$ (EGZB), being the department for geriatric medicine of the Charité university hospital in Berlin and the largest geriatric clinic in Germany. Altogether $\mathrm{n}=5,176$ single episodes were extracted from the clinical information system $(3,384$ female, 1,792 male, RehaDoc system). These were matched with the clinic's paper-based fall incident reports, which are filled in for every fall event, amounting to $\mathrm{n}=493$ within the study period. A fall is defined as an unexpected event during which a patient involuntarily comes to rest on the ground. This does not include events during which patients are lowered to the ground by staff members. The average age of the patients was 77.5 years, and their mean Barthel index score was 44.7 points ( $\mathrm{SD}=26.3$ points).

The research for this paper has been conducted in compliance with the Helsinki Declaration. The use of anonymized patient data for research has been approved by the ethics committee of the Charite university hospital, and a consent form to that effect is signed by every patient or her or his legal representative on admission.

As is common in clinical data sets, not all items are available for every patient. This is of course partly due to forgotten entries, but primarily to the fact that several assessment tests or sub-tests have not been performed with the patients, e.g. because they were physically not capable (e.g. for the Performance-Oriented Mobility Assessment [6]), lived in a nursing home (social status questionnaire) or were mentally so inconspicuous that they were not tested at all (Mini-Mental State Examination). The items included in the extracted data sets are shown in Table 1 along with the percentage of missing values.

All of the above- mentioned items were used for the data mining algorithms, yet only a subset of them actually appears in the models, due to inherent attribute selection processes of the employed algorithms. These are e.g. based on their ability to part subgroups of fallers and non-fallers using the information gain (C4.5 algorithm) [14].

\section{Classification model induction and evaluation}

We used two supervised machine learning algorithms to induce classification models, the C4.5 classification tree algorithm introduced by Quinlan [15] (minimum number of instances per leaf $=20$, confidence factor $=0.25$ ) and a logistic regression algorithm (maximum boosting iterations $=500$, cross-validation), as implemented in the Waikato Environment for Knowledge Analysis (WEKA, version 3.7.2) [14]. The classification tree on the one hand is comprehensible, as similar rules resp. diagnostic algorithms are well known among clinicians, and it allows for the extraction of explicit classification rules as well as high-risk subgroups within a population [11], both of which can be useful in clinical practice [16]. Logistic regression models on the other hand are known to be more stable than decision trees with regard to missing data and small changes in the data sets which often lead to changes in tree structure. For both algorithms the binary attribute fall (yes/no) was used as reference for the induction of the model. Missing values are treated by the two algorithms using different strategies: C4.5 splits the training data instances to the leaves of the decision tree proportionally to the occurrence of missing data in the data set. The logistic regression algorithm, in contrast, replaces missing values using the means of non-missing values in the training data set.

In order to optimize the models' predictive performance to serve as a screening test, a $2 \times 2$ cost matrix was employed, defining the relative cost of false negatives - patients who fall but are not identified by the model - as 20 -fold higher than those of false positives. 
Table 1 Items and item sets used to induce the classification models along with the percentages of missing values in our data set ( $n=5,176$ cases); the 19 Lachs and 22 Tinetti sub-scores are not listed separately Item (set) name Missing values in $\%$

\begin{tabular}{ll}
\hline Age on admission & 0.0 \\
\hline Sex $(\mathrm{m} / \mathrm{f})$ & 0.0 \\
\hline
\end{tabular}

Social status (35 sub-items concerning social contacts, 54.3 activities, living, economic situation)

\begin{tabular}{ll}
\hline Barthel index sum score [12] & 2.2 \\
\hline Lachs score (16 sub-items [13]) & $0.0-19.4$ \\
\hline Timed 'Up \& Go' test total time & 58.8 \\
\hline
\end{tabular}

Performance-Oriented Mobility Assessment (POMA) by 31.7-68.5 Tinetti (22 sub-items [6])

Mini-Mental State Examination (MMSE) score on 53.2 admission

\begin{tabular}{ll}
\hline Number of diagnoses on admission & 0.7 \\
\hline Number of different medications on admission & 1.0 \\
\hline Fall (yes/no) & 0.0 \\
\hline
\end{tabular}

This estimate is based on the consideration that false negatives are extremely costly. Despite several published studies on the overall cost of falls (e.g. [5,17]), only few studies exist which compare long-term costs of fallers and non-fallers. A recent study by Carroll et al. reports a difference of about 6200US\$ per person per year between those two groups [18]. Apart from the cost matrix, a classifier optimization algorithm called Threshold Selector [14] was used. It optimizes the F-measure, i. e. an established classification performance measure defined as

$$
\frac{2 * T P}{2 * T P+F P+F N}
$$

$(\mathrm{TP}=$ true positives, $\mathrm{FP}=$ false positives, $\mathrm{FN}=$ false negatives)

The evaluation of the classification models was done by means of a ten times ten-fold cross-validation, and performance was assessed by calculating sensitivity, specificity, positive and negative predictive values (PPV/ NPV), classification accuracy, the area under the curve (AUC) and the likelihood ratios of positive (+LR) and negative test results (-LR) along with their 95\% confidence intervals.

\section{Risk group identification}

In order to identify subgroups with a high in-patient fall risk, classification rules having the condition fall $=$ yes as the rule's consequent were read from the decision tree model. In this process, we only considered rules that were applicable to at least 100 instances from our data set - thus having an acceptable coverage -and which had a relative accuracy of at least $70 \%$.

\section{Results}

Table 2 shows the classification results of our decision tree model along with the contingency table. The decision tree itself is not included due to it size of 107 nodes. Following the rules of Good Scientific Practice (e. g. [19]), the complete classification tree may be requested from the first author. The results show that about two thirds (67.1\%) of non-fallers, but little more than half $(55.4 \%)$ of fallers are identified correctly. An NPV of $93.5 \%$ is pitted against a low $15 \%$ for PPV. The overall classification accuracy amounts to $66 \%$, accompanied by a poor 0.63 AUC value. The likelihood ratios (Table 3) confirm that the results differ from chance, but also that the test results enhance diagnostic accuracy only marginally [20].

Very similar results (Tables 4 and 3) are found by the logistic regression model. While the AUC and the NPV are equal to that of the decision tree, the classification accuracy (56.2\%), specificity (55.4\%), and PPV (13\%) are lower. Only the sensitivity (63.5\%) is higher. The + LR and -LR values (Table 3) are similar to that of the decision tree model.

Table 5 contains five classification rules representing high risk subgroups, all of which have been extracted with the consequent fall = yes, along with their relative accuracy within the data set.

\section{Discussion}

The classification results show that the classification models can only identify slightly more than half (55.4/ 63.5\%) of the patients who will suffer from a fall during their in-patient stay (aim\#1). This result is similar to those obtained e.g. by Kim et al. [10] or in the meta analysis performed by Oliver et al. [9], who conclude that even the best tools cannot identify a large majority of fallers. For some of these patients, a fall might be avoided, provided that effective preventive measures are taken in time. This potential benefit, however, is countered by low positive predictive values of just $15 / 13 \%$,

Table 2 Classification results and contingency table for our decision tree model $(n=5,176)$

\begin{tabular}{|c|c|c|c|c|c|}
\hline \multicolumn{2}{|l|}{ decision tree model } & & & & \\
\hline Classification accuracy & $66.0 \%$ & & & & \\
\hline sensitivity & $55.4 \%$ & & \multicolumn{2}{|c|}{ Prediction } & \\
\hline specificity & $67.1 \%$ & & No & yes & Sum \\
\hline neg. predictive value & $93.5 \%$ & no fall & 3141 & 1542 & 4684 \\
\hline pos. predictive value & $15.0 \%$ & Fall & 220 & 273 & 493 \\
\hline$\overline{A \cup C}$ & 0.63 & Sum & 3361 & 1815 & 5176 \\
\hline
\end{tabular}


Table $3+$ LR and -LR values of the classification models (decision tree and logistic regression) including their 95\% confidence intervals ( $n=5,176$ )

\begin{tabular}{lcl}
\hline model name & +LR value $(\mathbf{9 5 \%} \mathbf{C l})$ & - LR value $(\mathbf{9 5 \%} \mathrm{Cl})$ \\
\hline decision tree & $1.68(1.54-1.84)$ & $0.67(0.6-0.74)$ \\
\hline logistic regression & $1.43(1.32-1.53)$ & $0.66(0.59-0.74)$ \\
\hline
\end{tabular}

making this approach costly and thus rendering it useless. The negative predictive value, in turn, is high in both models (93.5\%), so that patients who will not fall and therefore do not need specific preventive measures - can be identified correctly. Overall, the results are similar to those obtained in a previous smaller study conducted by some of the authors [21], and they seem disappointing, especially as the test battery contains established and validated tests often used for assessing fall risk, such as the Timed Up \& Go [22] or the POMA [6]. On the other hand, a high fall risk is not necessarily associated with an actual fall event which to some extent is random in a short and variable in-patient period of time, even more so if a special environment such as a geriatric ward is the setting. As such, our model very likely suffers from a multitude of influencing factors (e.g. post-operative weakness, unfamiliar environment, problems with sleeping, analgesia medication), part of which are neither assessed during an in-patient stay, nor are controllable.

A closer analysis of the rules defining the high-risk subgroups from the data sets of the 493 fall incidents reveals a number of factors which are associated with a higher than normal risk and are also found in literature as well as are part of experiential clinical knowledge. First of all, an age above 70 years obviously can be regarded as a risk factor, as can a Barthel index score of $\leq 45$ pts. The latter reveals a significant limitation in a person's overall ability to cope with daily life, such as toileting or mobility [12]. Old age of course is attributed to frailty, originating e.g. either from sarcopenia or the existence of co-morbidity concerning chronic diseases such as e.g. arthritis or diabetes. Steinhagen-Thiessen and Borchelt e.g. report results from the Berlin Aging Study showing that $88 \%$ of the persons aged 70 years or

Table 4 Classification results and contingency table for our logistic regression model $(n=5,176)$

\begin{tabular}{|c|c|c|c|c|c|}
\hline \multicolumn{2}{|c|}{ logistic regression model } & & & & \\
\hline \multicolumn{2}{|c|}{ Classification accuracy $\quad 56.2 \%$} & & & & \\
\hline sensitivity & $63.5 \%$ & & \multicolumn{2}{|c|}{ Prediction } & \\
\hline specificity & $55.4 \%$ & & No & yes & Sum \\
\hline neg. predictive value & $93.5 \%$ & no fall & 2596 & 2087 & 4684 \\
\hline pos. predictive value & $13.0 \%$ & Fall & 180 & 313 & 493 \\
\hline$\overline{A \cup C}$ & 0.63 & Sum & 2776 & 2400 & 5176 \\
\hline
\end{tabular}

Table 5 Classification rules extracted from the decision tree model; only rules with the condition fall $=$ yes as consequent and which cover a number of at least 100 instances and have a related accuracy of at least $70 \%$ were considered; the rules are ordered by their relative accuracy

\begin{tabular}{|c|c|c|}
\hline no. & rule (consequent: fall=yes) & $\begin{array}{l}\text { relative } \\
\text { accuracy }\end{array}$ \\
\hline $\begin{array}{l}1 a \\
b\end{array}$ & $\begin{array}{l}\text { (Barthel index score } \leq 45 \mathrm{pts} \text { ) and (sex = male) and } \\
\text { (age }>75 \mathrm{y}) \\
\text { and (Lachs depression item }=0 \text { ) }\end{array}$ & $\begin{array}{l}84.3 \% \\
77.8 \%\end{array}$ \\
\hline 2 & $\begin{array}{l}\text { (Barthel index score }>10 \text { and } \leq 45 \text { pts) and (sex = } \\
\text { female) and (number of medications }<14 \text { ) and } \\
\text { (MMSE score } \leq 26 \text { pts) and (institutionalized=yes) } \\
\text { and (needs aid for standing) }\end{array}$ & $80.0 \%$ \\
\hline 3 & $\begin{array}{l}\text { (Barthel index score }>45 \text { and } \leq 65 \mathrm{pts} \text { ) and (MMSE } \\
\text { score }>21 \mathrm{pts} \text { ) and (Timed 'Up\& Go' time } \leq 42 \mathrm{~s} \text { ) } \\
\text { and (number of diagnoses }>11 \text { ) and (number of } \\
\text { medications }>8 \text { ) }\end{array}$ & $72.8 \%$ \\
\hline 4 & $\begin{array}{l}\text { (Barthel index score }>45 \text { and } \leq 65 \text { pts) and (MMSE } \\
\text { score } \leq 18 \text { pts) }\end{array}$ & $72.1 \%$ \\
\hline 5 & $\begin{array}{l}\text { (Barthel index score }>45 \text { and } \leq 65 \text { pts) and (MMSE } \\
\text { score }>18 \text { pts) and (Timed 'Up\& Go' time }>42 \mathrm{~s} \text { ) } \\
\text { and (age }>71 \mathrm{y} \text { ) }\end{array}$ & $71.9 \%$ \\
\hline
\end{tabular}

above suffer from at least five somatic diseases [23]. Cognitive impairment as defined by a low MMSE score also constitutes a risk for fall events in geriatric patients [24]. In addition to this, being institutionalized represents a risk, but this result is likely influenced by a negative selection bias, as people are often admitted to institutions because they have become too weak to live independently and for this reason may have an elevated risk [1]. Finally, a high degree of co-morbidity as well as polypharmacy is attributed with a high risk of falling. The latter confirms results reported e.g. by Kojima et al. [25] or Chang et al. [26], and questions asking for certain psychotropic medications are part of e.g. the STRATIFY score [8].

Along with risk group identification, we have to look at therapeutic consequences of identifying potential fallers and predicting in-patient falls. Although we inherently hypothesize that, if we predict these events correctly, we will be able to initiate preventive measures that will avoid at least a certain proportion of fall incidents, we cannot prove this until a sound controlled study has been performed. Also, if viewed from an economic perspective, we currently do not know if the benefit of in-patient fall risk screening and especially the following interventions outweigh the costs of such an endeavor.

\section{Limitations}

Classification trees tend to be unstable in small data sets. Therefore we have also used a logistic regression model in addition. Nevertheless, according to our aim\#2 
to identify high-risk subgroups, we deliberately chose the decision tree approach [11] despite our mediumsized data set ( $>5,000$ instances). Furthermore, we expected - and found - non-linear relationships for some parameters (e.g. age), confirming the justification of our choice. The significant amount of missing data for some sub-items limits the generalizability of our findings, yet this is quite normal in clinical data sets where it is often neither necessary nor practical to apply all available test procedures. We have used model induction algorithms that employ two different strategies of dealing with missing data, thus minimizing the effect. Finally, the cost matrix defining the costs of false negatives as being 20-fold higher than those of false positives, is a rough estimate as mentioned above and therefore is to some extent arbitrary, as the authors are not aware of an explicit study providing a ratio comparing long-term in-patient fall-related costs with those of non-fallers.

\section{Conclusion}

Based on more than 5,000 data sets obtained from a clinical data base of a geriatric hospital, we generated two classification models that are able to detect 55.4/ $63.5 \%$ of fallers and $67.1 / 55.4 \%$ of non-fallers correctly. Furthermore, we identified five subgroups with a high risk of falling during an in-patient stay. The description of these groups and the interpretation of the risk factors found (age, low ADL score, cognitive impairment, institutionalization, polypharmacy and co-morbidity) may be useful in future practice for screening geriatric patients on admission to a hospital for their individual risk. Our future work will include the generation of a new hybrid risk classification model that incorporates both medical domain knowledge as well as the knowledge gained from our data mining approach. Such a model could be updated repeatedly with new data, enabling its customization for different populations of patients or even different hospital environments. Finally, further research work is needed to evaluate our models in a prospective controlled setting as well as from an economic perspective.

\section{Acknowledgements \\ This publication is supported by the project "Open Access Publizieren" by the Deutsche Forschungsgemeinschaft (DFG). We thank the members of the computer science group at the Department of Geriatric Medicine (Charité University Medicine, Berlin) for extracting the data sets necessary for this research work.}

\section{Author details}

'Peter L. Reichertz Institute for Medical Informatics, University of Braunschweig - Institute of Technology and Hanover Medical School, CarlNeuberg-Str. 1, 30625 Hanover, Germany. ${ }^{2}$ Peter L. Reichertz Institute for Medical Informatics, University of Braunschweig - Institute of Technology and Hanover Medical School, Mühlenpfordtstr. 23, 38106 Braunschweig,
Germany. ${ }^{3}$ Geriatrics Research Group, Department of Geriatric Medicine, Charité University Medicine, Reinickendorfer Str. 61, 13347 Berlin, Germany.

\section{Authors' contributions}

MM supervised the study, carried out the data analysis and drafted the manuscript. MG organized the data export from the hospital information system and interpreted the results of the analysis. SR preprocessed the data sets and conducted part of the data analysis. MGie provided statistical and methodological feedback for the analysis. MS participated in the data analysis and the computation of performance values. KHW participated in drafting the study protocol. EST conceived of the study and participated in its coordination. All authors read and approved the final manuscript.

\section{Competing interests}

The authors declare that they have no competing interests.

Received: 14 June 2011 Accepted: 14 March 2012

Published: 14 March 2012

\section{References}

1. Heinze C, Halfens RJ, Dassen T: Falls in German in-patients and residents over 65 years of age. J Clin Nurs 2007, 16:495-501.

2. Kannus $P$, Sievanen $H$, Palvanen M, Jarvinen T, Parkkari J: Prevention of falls and consequent injuries in elderly people. Lancet 2005, 366:1885-1893.

3. Salva A, Bolibar I, Pera G, Arias C: Incidence and consequences of falls among elderly people living in the community. Med Clin (Barc) 2004, 122:172-176.

4. Tinetti ME, Mendes CFde Leon, Doucette JT, Baker DI: Fear of falling and fall-related efficacy in relationship to functioning among communityliving elders. J Gerontol 1994, 49:M140-M147.

5. Stevens JA, Corso PS, Finkelstein EA, Miller TR: The costs of fatal and nonfatal falls among older adults. Inj Prev 2006, 12:290-295.

6. Tinetti ME: Performance-oriented assessment of mobility problems in elderly patients. J Am Geriatr Soc 1986, 34:119-126.

7. Gates S, Smith LA, Fisher JD, Lamb SE: Systematic review of accuracy of screening instruments for predicting fall risk among independently living older adults. J Rehabil Res Dev 2008, 45:1105-1116.

8. Oliver D, Britton M, Seed P, Martin FC, Hopper AH: Development and evaluation of evidence based risk assessment tool (STRATIFY) to predict which elderly inpatients will fall: case-control and cohort studies. BMJ 1997, 315:1049-1053.

9. Oliver D, Papaioannou A, Giangregorio L, Thabane L, Reizgys K, Foster G: A systematic review and meta-analysis of studies using the STRATIFY tool for prediction of falls in hospital patients: how well does it work? Age Ageing 2008, 37:621-627.

10. Kim EA, Mordiffi SZ, Bee WH, Devi K, Evans D: Evaluation of three fall-risk assessment tools in an acute care setting. J Adv Nurs 2007, 60:427-435.

11. de Rooij SE, Abu-Hanna A, Levi M, de Jonge E: Identification of high-risk subgroups in very elderly intensive care unit patients. Critical Care 2007, 11(2):R33.

12. Mahoney Fl, Barthel DW: Functional Evaluation: The Barthel Index. Md State Med J 1965, 14:61-65.

13. Lachs MS, Feinstein AR, Cooney LM Jr, Drickamer MA, Marottoli RA, Pannill FC, Tinetti ME: A simple procedure for general screening for functional disability in elderly patients. Ann Intern Med 1990, 112:699-706.

14. Witten IH, Frank E: Data Mining: Practical Machine Learning Tools and Techniques, Second Edition (Morgan Kaufmann Series in Data Management Systems) Morgan Kaufmann Publishers Inc; 2005.

15. Quinlan JR: C4.5: Programs for machine learning San Francisco: Morgan Kaufman; 1993.

16. Stollhoff R, Sauerbrei W, Schumacher M: An experimental evaluation of boosting methods for classification. Methods Inf Med 2010, 49:219-229.

17. King MB, Tinetti ME: Falls in community-dwelling older persons. J Am Geriatr Soc 1995, 43:1146-1154.

18. Carroll NV, Delafuente JC, Cox FM, Narayanan S: Fall-related hospitalization and facility costs among residents of institutions providing long-term care. Gerontologist 2008, 48:213-222.

19. Proposals for Safeguarding Good Scientific Practice 1998. [http://www. dfg.de/aktuelles_presse/reden_stellungnahmen/download/ self_regulation_98.pdf]. 
20. Jaeschke R, Guyatt GH, Sackett DL: Users' guides to the medical literature. III. How to use an article about a diagnostic test. B. What are the results and will they help me in caring for my patients? The Evidence-Based Medicine Working Group. Jama 1994, 271:703-707.

21. Marschollek M, Nemitz G, Gietzelt M, Wolf KH, Meyer Zu Schwabedissen H, Haux R: Predicting in-patient falls in a geriatric clinic: a clinical study combining assessment data and simple sensory gait measurements. $Z$ Gerontol Geriatr 2009, 42:317-321.

22. Podsiadlo D, Richardson S: The timed "Up \& Go": a test of basic functional mobility for frail elderly persons. J Am Geriatr Soc 1991, 39:142-148.

23. Steinhagen-Thiessen E, Borchelt M: Morbidität, Medikation und Funktionalität im Alter [Morbidity, medication and functional status in the elderly]. In Die Berliner Altersstudie [The Berlin Aging Study]. Edited by: Mayer KU, Baltes PB. Berlin: Akademie Verlag; 1996:151-184.

24. Vieira ER, Freund-Heritage $R$, da Costa BR: Risk factors for geriatric patient falls in rehabilitation hospital settings: a systematic review. Clin Rehabil 2011, 25(9):788-799.

25. Kojima T, Akishita M, Nakamura T, Nomura K, Ogawa S, lijima K, Eto M Ouchi Y: Association of polypharmacy with fall risk among geriatric outpatients. Geriatr Gerontol Int 2011, 11(4):438-444.

26. Chang CM, Chen MJ, Tsai CY, Ho LH, Hsieh HL, Chau YL, Liu CY: Medical conditions and medications as risk factors of falls in the inpatient older people: a case-control study. Int J Geriatr Psychiatry 2011, 26:602-607.

\section{Pre-publication history}

The pre-publication history for this paper can be accessed here: http://www.biomedcentral.com/1472-6947/12/19/prepub

doi:10.1186/1472-6947-12-19

Cite this article as: Marschollek et al:: Mining geriatric assessment data for in-patient fall prediction models and high-risk subgroups. BMC

Medical Informatics and Decision Making 2012 12:19.

\section{Submit your next manuscript to BioMed Central and take full advantage of:}

- Convenient online submission

- Thorough peer review

- No space constraints or color figure charges

- Immediate publication on acceptance

- Inclusion in PubMed, CAS, Scopus and Google Scholar

- Research which is freely available for redistribution

Submit your manuscript at www.biomedcentral.com/submit 